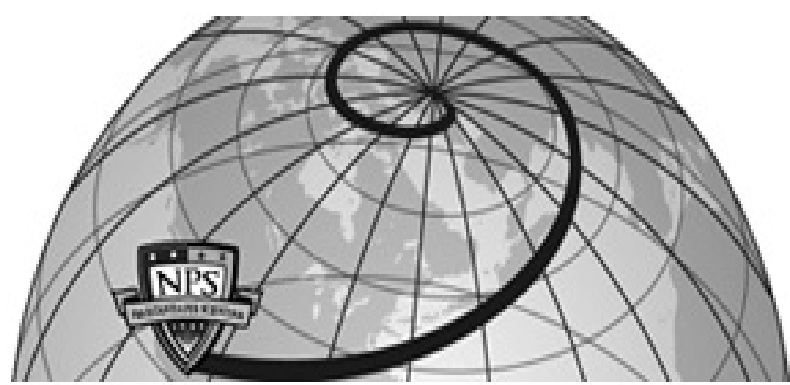

Calhoun: The NPS Institutional Archive DSpace Repository

Nonparametric Statistical Analysis of the Reliability of a Naval Aviation Propulsion System

Millar, Richard C.; Mazzuchi, Thomas Andrew; Sarkani, Shahram

Naval Engineers Journal

https://hdl.handle.net/10945/43486

This publication is a work of the U.S. Government as defined in Title 17, United States Code, Section 101. Copyright protection is not available for this work in the United States.

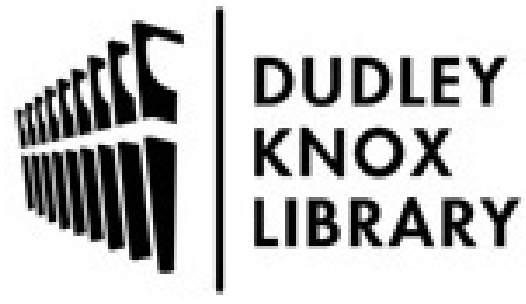

http://www.nps.edu/library
Calhoun is the Naval Postgraduate School's public access digital repository for research materials and institutional publications created by the NPS community. Calhoun is named for Professor of Mathematics Guy K. Calhoun, NPS's first appointed -- and published -- scholarly author.

Dudley Knox Library / Naval Postgraduate School 411 Dyer Road / 1 University Circle Monterey, California USA 93943 


\title{
Nonparametric Statistical Analysis of the Reliability of a Naval Aviation Propulsion System
}

Richard C. Millar, Thomas Andrew Mazzuchi, and Shahram Sarkani

\begin{abstract}
Hbstract
This paper reports the results of applying lifetime (or reliability database) statistical analysis methods to engine removal data recorded over the initial 8 years' service of the General Electric F414-GE-400 turbofan engines propelling the F/A-18 E/F Super Hornet in service with the US Navy, with the intent of better understanding the reasons for engine removal and their impact on engine time on wing (TOW), and to gain an insight into the reliability of the modules and components from which the engine is assembled. It was found that "coloring" the engine removals into three classes of reasons for removal enabled lifetime data analysis revealing interesting and useful features of in-service engine reliability. Nonparametric statistical analysis provided actionable information on engine removal probability as a function of TOW and removal cause that should be applicable to planning flight operations, line maintenance, and support logistics. The analysis of engine removals due to hot section distress appears to disconfirm the presumption of independence between the three classes of removal. Opportunistic maintenance of modules made accessible due to engine removals to service other modules may significantly affect the observed engine removal distribution of the module of interest, i.e., competing risk masks the underlying module hazard functions.
\end{abstract}

\section{Introduction}

The General Electric Aviation (GE) F414-GE400 engine is the low-bypass gas turbine propulsion system installed in the F/A-18E/F Super Hornet (manufactured by the Boeing Company), a twin engine all-weather strike aircraft that entered service with the US Navy in 1999.

Deliveries of F414-powered F/A-18E/F Super Hornets from Boeing continue to this day, and so the engines in service span a wide range of calendar lives and total flight times. Some have never been removed from the aircraft they were delivered with; others have been removed for

The material presented in this paper has been approved by NAVAIR for public release, distribution unlimited, under SPR-07-343.265, SPR-07-449.265, SPR-07-533265, SPR-07-538-265, and SPR 08-045.265. maintenance and reinstalled in (other) aircraft up to nine times.

The F/A-18E/F aircraft is typically carrier based for operations and, as a consequence, the maintenance philosophy and process applied to all subsystems is tailored to the demanding shipboard environment, with its restricted space, limited manpower, and challenging logistics.

The F414-GE-400 is maintained using a military maintenance process with three organizational levels: the O-Level (operational), I-Level (intermediate), and D-Level (depot). This maintenance structure is well suited to the shipboard operational context, and the design and the configuration of the F414 is modular (Figure 1) to allow engine refurbishing and 
Figure 1: F414GE-400 Modules (C)2007 General Electric Company, accessible at $h$ ttp:// www.geae.com/ engines/military/ f414/maintenance. $\mathrm{html}$; reproduction and use governed by the terms at http:// www.ge.com/ terms.html)

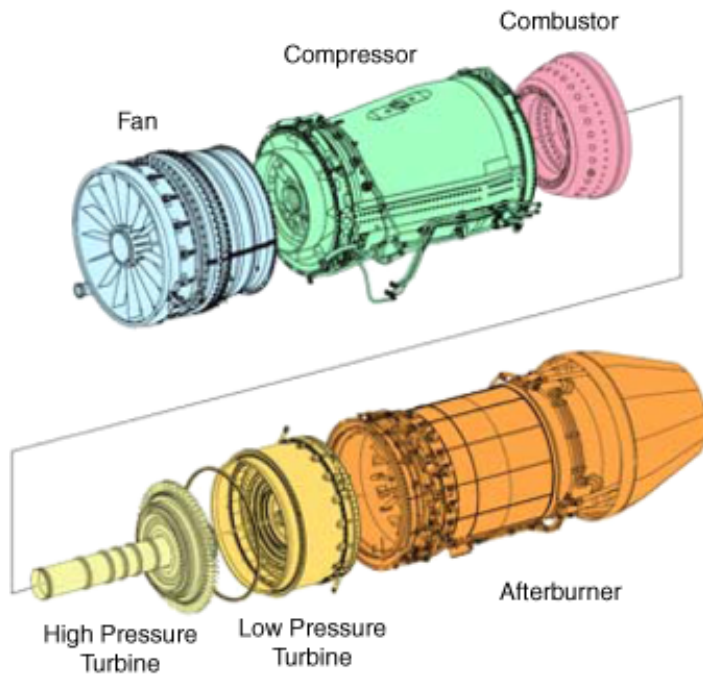

rebuilding by changing out only the module(s) affected by the reason why the engine was removed from the aircraft at the O-Level (base or shipboard). The fan section, high-pressure compressor, combustor, high-pressure turbine, lowpressure turbine, and afterburner are separately tracked as modules that can be independently removed at the I-Level for depot maintenance. (The F414 is a two-shaft engine; the low-pressure turbine drives the fan while the highpressure turbine independently drives the highpressure compressor.)

Maintenance activity at the O-Level is limited to regular external inspections and servicing, troubleshooting flight crew "squawks," fault indications and functional failures, periodic inspection for internal distress or other inspections intended to preempt specific component failure modes, replacement of an externally accessible weapons replaceable article (WRA), and removal of the complete F414 engine unit. (The periodic inspection occurs at a preventive maintenance interval of about 0.6 engine flight hour $[\mathrm{EFH}]$ index-the usage parameter used as a measure of lifetime in the following analysis.) Engine removals may be "scheduled" for planned preventive maintenance, including mandatory replacement "life-limited" components and inspections of high time components, or "unscheduled" due to unanticipated failure or unacceptable performance degradation. A large proportion of unscheduled removals are the result of deficiencies discovered during the periodic O-Level inspection for internal distress.

The engines removed are shipped to a shorebased I-Level facility for detailed inspection and assessment to isolate the failure and secondary damage to one or more engine modules. If the affected engine modules cannot be made serviceable at the I-Level, the engine is broken down and the affected modules are shipped to the F414 depot for repair. The engine is then rebuilt by drawing on the available pool of available spare modules, taking into consideration the remaining scheduled useful life of the modules (and their component parts) to accommodate a target time on wing (TOW) before the next scheduled removal. Schoch (2003) provides a more detailed description of the maintenance process for the F414-GE-400.

Early in the work reported here, it was decided to focus on the O-Level maintenance process for the F414 engine and specifically on engine removals as the most costly, operationally disruptive, and logistically challenging events recorded. The primary intent of the analysis is to understand the determinants and characteristics of F414 engine removals to enable improved operational, maintenance, and logistics planning.

It was hoped that the above F414 maintenance process would also allow an insight into module and component reliability as a function of usage (TOW). A fundamental assumption was that there were no significant deviations from the modular maintenance process described above, i.e., modules were only subject to refurbishment or replacement if implicated in the reason for removal cited in the maintenance records.

The data source for this analysis is one of the most advanced reliability databases (RDB) used in support of naval aviation weapon systems. This RDB, designated by General Electric as their "Military Data Warehouse" (MDW), tracks the usage and reliability of the 
F414-GE-400. GE compiles and maintains the F414 MDW using data available from US Navy sources, with event scoring and supplemental entries by GE technical support personnel.

A primary function of the MDW RDB is to provide an overview of F414 safety, reliability, and readiness, predominantly trends for the total fleet versus calendar time. These calendar-based trends necessarily track a changing aircraft population, because deliveries of new F/A-18 E/F aircraft have been continuous and unabated since its introduction in 1999. (The evolving age distribution of the fleet may influence the observed removal rates if the failure distributions significantly differ from exponential distributions, i.e., constant hazard rates.)

The critical high-level metrics currently monitored to assess fleetwide engine health and reliability are unscheduled engine removal (UER) rates, engine TOW, ground and flight abort rates, and in-flight shutdown rates (IFSD). These are calculated monthly as fleet averages, tracked versus cause and reason for removal, and absolute levels and trends are monitored versus calendar time.

The MDW also supports tracking of life-limited components, components that must be replaced at a predetermined usage level based on EFHs, operating cycles, other life usage indicators, and combinations of more than one usage parameter. Such "hard-time" component removals usually result in a scheduled O-Level removal of the engine and its return to an I-Level station for removal and replacement of the module containing the time-expired component, which is then delivered to the F414 depot for teardown, component replacement, rebuilding, and test.

The third use of the MDW, identifying, assessing, and mitigating emerging safety and readiness issues, is less visible as it typically requires customized analysis of the available MDW data. Leading reliability degraders are identified by Pareto analysis, and the reliability of specific components may be assessed using parametric models for lifetime data, typically Weibull distributions.

The MDW contains extensive records of maintenance activity and reliability at the module, WRA, and serialized component level, but these were not investigated in the analysis reported here, which was limited in scope to the O-Level removals.

The work reported here applied statistical methods for lifetime data analysis, also known as RDB analysis, to UER at O-Level, to better understand the evolution and outcomes of the total process of managing the reliability of propulsion systems in Naval aviation service. It was understood that this investigation would provide only indirect information on the underlying (latent) module removal probabilities.

For example, scheduled engine removals (SER) are an "independent" variable, in the sense that they are preventive maintenance that is part of the maintenance and configuration management process. SER that affect the reliability of the affected module (their intended purpose in most cases) will mask the latent module removal probabilities, i.e., SER are a likely source of competing risk. (See Lawless 2003 for a recent treatment of the difficulties introduced by competing risks.)

This is a radical departure from the roots of lifetime data analysis in medical and materials research. In-service aircraft engines are not a random sample of nominally identical items treated to carefully defined and controlled protocols. Over the 8 years of records extracted from the GE F414 RDB, engine configuration, and maintenance procedures were actively modified in response to the observed reliability of the engines.

Operational aircraft availability and costs are strongly influenced by engine reliability, and so any shortcomings from expected and tolerable reliability levels were, and are, subject to 
aggressive mitigation to restore fleet capabilities. Furthermore, high time engines (and modules) removed for scheduled or unscheduled maintenance are proactively inspected for signs of distress or incipient failure. Significant findings are likely to result in corrective action.

Thus, in many ways, the MDW lifetime data analysis presented here has more affinity with actuarial or epidemiological studies, where evolving differences in the population studied and relevant interventions are crucially important in analyzing and understanding the lifetime data. However, more so than in either of these fields, the purpose of this study is to provide tools to improve the timeliness and effectiveness of "closed-loop" control of the outcomes, in this case effective propulsion system reliability in the field in the context of an intensive preventive maintenance process. It was hoped that this analysis would yield qualitative insights into module reliability, particularly its evolution with changing engine configuration and other interventions.

\section{Hnalysis of F414 Engine Removals}

Note: In all that follows, the F414-GE-400 EFHs reported by the GE MDW were scaled by a constant, to protect proprietary and controlled information on F/A-18E/F operations and F414 availability. Thus, accumulated operational life in $\mathrm{EFH}$ and time since new (TSN) and time since removal (TSR) data are all presented as EFH Indices, TSN Indices, and TSR Indices, denominated by a more or less arbitrary EFH figure. (TSN is a basic metric for removals-the accumulated EFH count since the engine was first installed in an aircraft. TSR is the accumulated EFH since the latest prior removal of the engine.)

October 2006 was taken as a baseline for this analysis, and full records of all engine removals from entry into service to this date were acquired. This file records 1,174 engine removal events from May 1999 to October 2006. Each event record included 68 entries, including dates, locations and organizations, aircraft and engine position, engine, module and part serial numbers and identity, control numbers, symptoms and reason for removal, field and shop findings and remarks, maintenance labor hours and duration, links to maintenance documentation, a variety of codes-unplanned versus planned removals, flight and ground abort, IFSD, etc.-and time (in $\mathrm{EFH}$ ) since new (TSN), and since the previous removal (TSR).

For the purposes of this research, this engine removals database was extensively edited to remove sensitive information and detail that impeded analysis. Only records from F/A-18 E/F operational service (exclusive of developmental flight test) were retained, as exemplified by the extract shown in Table 1. This consists of a reduced data set for each removal: an identifier unique to each engine derived from the sequential production serial numbers, a reason for removal code for unscheduled removals (defined below), counters for scheduled removals versus unscheduled removals and ground and flight aborts, and IFSD, and TSR and TSN indices.

The many SER (indicated by SER Count $=1$ in Table 1) presented a quandary; although UER were clearly most relevant to the task at hand, SER are intended to preempt UER, affecting the operational outcomes, and may thus represent a competing risk for UER. Also, an SER will "censor" the removal data for any reason for removal code where the associated configuration changes modify the probability of future removals for that code, possibly "renewing" the engine for that code. In the analysis presented here this possibility was effectively neglected. Furthermore, the SER requirements are dynamic; they are deliberately modified over time in response to observed engine reliability. Nevertheless, it was pragmatically decided to ignore SER for the following analysis and return to these issues in light of the findings on UER. Further study is planned in future work that will access detailed data from I-Level and depot maintenance records in the GE MDW RDB, which will hopefully allow a better accounting of censoring and competing risk impacts of SER at the component and module level. 


\section{TABLE 1: Structure of edited engine removals Database}

\begin{tabular}{|c|c|c|c|c|c|c|c|c|}
\hline $\begin{array}{l}\text { Engine } \\
\text { Identifier }\end{array}$ & $\begin{array}{c}\text { R for R Count } \\
1=\text { FOD, } \\
2=\text { Comb ... }\end{array}$ & $\begin{array}{l}\text { UER } \\
\text { Count }\end{array}$ & $\begin{array}{l}\text { SER } \\
\text { Count }\end{array}$ & $\begin{array}{c}\text { Ground } \\
\text { Abort } \\
\text { Count }\end{array}$ & $\begin{array}{l}\text { Flight } \\
\text { Abort } \\
\text { Count }\end{array}$ & $\begin{array}{l}\text { In-Flight } \\
\text { Shutdown } \\
\text { Count }\end{array}$ & $\begin{array}{c}\text { TSR } \\
\text { Index }\end{array}$ & $\begin{array}{c}\text { TSN } \\
\text { Index }\end{array}$ \\
\hline 1 & & 0 & 1 & 0 & 0 & 0 & 0.7237 & 0.81 \\
\hline 1 & 5 & 1 & 0 & 1 & 0 & 0 & 0.1532 & 0.96 \\
\hline 1 & 3 & 1 & 0 & 0 & 1 & 0 & 0.4565 & 1.42 \\
\hline 1 & 0 & 1 & 0 & 0 & 1 & 0 & 0.0180 & 1.44 \\
\hline 1 & & 0 & 1 & 0 & 0 & 0 & 0.9009 & 2.34 \\
\hline 1 & & 0 & 1 & 0 & 0 & 0 & 1.7778 & 4.12 \\
\hline 2 & & 0 & 1 & 0 & 0 & 0 & 0.7568 & 0.76 \\
\hline 2 & & 0 & 1 & 0 & 0 & 0 & 0.0931 & 0.85 \\
\hline 2 & 0 & 1 & 0 & 0 & 0 & 0 & 0.4324 & 1.28 \\
\hline 2 & & 0 & 1 & 0 & 0 & 0 & 0.6276 & 1.91 \\
\hline 2 & 4 & 1 & 0 & 0 & 0 & 0 & 1.5586 & 3.47 \\
\hline 2 & 5 & 1 & 0 & 0 & 0 & 0 & 1.6577 & 5.16 \\
\hline
\end{tabular}

The results presented here are an indicator of the combined effects of inherent component reliability, changes to maintenance procedures (such as SER intervals and requirements), and evolution of the engines' configuration over the period covered by this study on the engine removal rates for specific reasons. A rationale for this approach is that the objective of this research was not to measure the underlying "latent" reliability of the modules and components; the purpose is to demonstrate improved indicators of the effectiveness of the overall engine life management process.

The F414 MDW RDB included identification of the module charged with the removal at the OLevel, plus extensive findings at the I-Level, and the depot-plus comments from the GE service engineers. After considerable study, this information was summarized as eight reasons for removal codes defined specifically for this study, as shown in Table 2. These combine distinct competing failure and degradation modes within the module (or class of failure) affected, while attempting to segregate modes driven by differing root causes-usage versus external events versus maintenance induced.

In most cases, the above categories follow the basic engine module breakdown fan, compressor, combustor, high-pressure turbine, low- pressure turbine, and afterburner, with Code 5 for oil and fuel leaks and externals, including the accessory gearbox and fuel system, oil system, and control system components.

In the case of the compressor, it was necessary to distinguish between intrinsic component failures (Code 7) and those with external causesspecifically damage due to foreign object ingestion damage (FOD) — which was assigned to Code 1.

There were no indications of internal compressor failures driving engines "off wing" prematurely. Code 7 removals were not associated with compressor turbo-machinery; they were predominantly due to failures in the external linkages and levers controlling compressor variable geometry.

The reason for removal Code 0 cuts across all modules and was assigned when removal was seen to be due to maintenance error or other mishaps, including cases where no cause could be identified or the reported cause could not be confirmed at the I-Level. Here it was also decided to include the small number of removals for miscellaneous reasons that did not fit the intent of other codes.

These codes were defined to suit the intent to isolate subsets of the removals with distinctive failure 


\begin{tabular}{ll} 
TABLE 2: & Reason for Removal Codes \\
Used for Analysis \\
\hline Code 0 & Mishaps and maintenance errors \\
\hline Code 1 & Foreign object damage (FOD) \\
\hline Code 2 & Combustor \\
\hline Code 3 & High-pressure turbine \\
\hline Code 4 & Low-pressure turbine \\
\hline Code 5 & $\begin{array}{l}\text { Externals and subsystems } \\
\text { (e.g., control, fuel, and lubrication systems) }\end{array}$ \\
\hline Code 6 & Afterburner \\
\hline Code 7 & Fan and compressor mechanical (intrinsic) \\
\hline
\end{tabular}

distributions driven by differing usage and exposure factors. For example, turbine and combustor module degradation and failures are driven by thermomechanical stresses (cycling from low to high power and speed) and material creep and degradation (time at high power and speed). To a first-order approximation, these factors should be related to component and engine service time and flight cycles. FOD and maintenance error might be expected to be more random, i.e., a constant hazard throughout the engine's service life.

Of the 665 recorded unscheduled removals, as detailed in Figure 2, most were accounted for by four primary and distinct causes, and Codes 2, 3, and 4 were all related to the thermomechanically stressed engine hot section. Code 6 could be eliminated at little cost, as the afterburner is quite distinct from the basic engine. As analysis proceeded, it became evident that the reasons for removal 0,5 , and 7 might be combined for economy of analysis, due to similarities in the statistical characteristics of the data and the common factor of being external to the core engine and vulnerable to maintenance and other exogenous effects.

The resulting three classes of UER (FOD, core engine distress, and residual, external, reasons) were then subjected to a lifetime statistical analysis, to provide a distinct and hopefully informative view of engine reliability between fleet-wide averages and rates and detailed module and component RDB analysis. Before moving on to the method developed to do this, there are issues to be addressed.

\section{Competing Risks and Censoring}

Given the complexity of a gas turbine engine and the maintenance process, competing risk needs to be considered. Within a given reason for removal code, different failure modes compete to drive a removal, but again this is only salient if we are attempting to understand and model the failure modes to predict module reliability. Because the scope of this analysis did not address the root cause of the removal, beyond confirmation of the reason for removal code from the information in the engine removal record, there was no consideration of competing risk at this level of granularity.

In ongoing and planned future work on GE MDW I-Level and depot maintenance data addressing module and component reliability, it should be possible to narrow the scope of competing risk to the serialized component level, and possibly within a component if parts replacements are recorded. Interesting issues will arise, such as the qualitative difference between a component failure and a removal of the same part number due to inspection at I-Level or depot, which might require estimating the failure time of the latter, or treating these as separate events and comparing the hazard functions. However, the ambiguities at the module and engine removal level should be minimized.

More salient to this research, it is a known fact that when the engine is at the I-Level to remove and replace one module, it may be decided to remove and replace another as preventive maintenance, a competing risk in the context of the reliability of the affected module. This could be due to shop inspection picking up a problem not noted at the O-Level, secondary damage, or the module might have scheduled maintenance due within the prescribed engine "build window" for delivery back to the fleet.

These opportunistic module removals, which should be considered as censoring, are not evident in the engine removals data studied here but may have a significant effect on the validity of 
the results, similar to SER that affect subsequent engine removal probabilities. The F414 MDW does provide this information in module-level reports and engine configuration records, and this will be accounted for in planned analyses of module and component reliability. However, as noted above for SER, and if our concern is characterizing engine removals, these effects are part of the total maintenance and configuration management process resulting in the engine removal outcomes we are attempting to assess.

Future work is planned to explain and model engine removals by extracting latent module and component reliability and building a model of overall reliability encompassing engine removals. This is beyond the scope of the research reported here. At this level, lifetime statistical methods accounting for censoring must be considered to counter the distortion of apparent engine reliability and calendar-based trends by the nonuniform ages of the engine population.

However, the engine removals data also require consideration of censoring. In looking into the data, it is evident that many engines were not removed during the time period covered, or were removed but not for the specific reasons for removal being considered in analyzing the data for a specific removals code. This negative evidence needs to be considered in arriving at a valid picture of engine reliability. See Lawless (2003) for an accessible treatment of the concept of censoring and the methods used here.

Since the removals data covered the period from initial F/A-18E/F fleet service up to October 2006, further analysis requires at least the EFHs since new (TSN) of all engines currently installed in aircraft or held as spares at the beginning of that month. These data were provided by a standard MDW report and are summarized as a histogram in Figure 3.

The total population of active engines was 620 . The TSN Index distribution in Figure 3 is a consequence of the operational tempo over the 8 -year service period studied, but the shape is

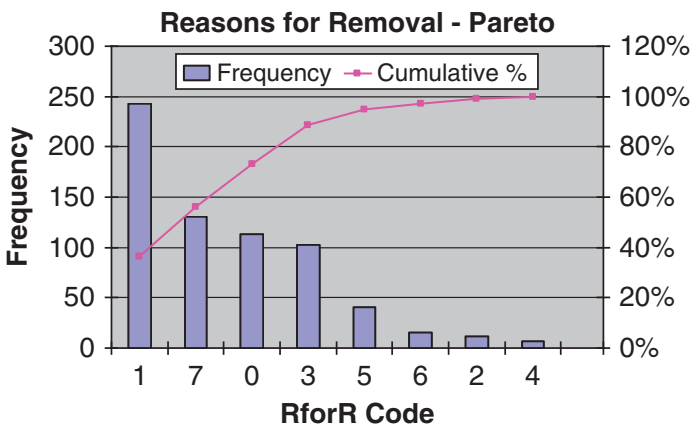

Figure 2: Reasons for Removal Pareto Analysis dominated by the effect of continued engine deliveries in new aircraft and spares provisioning throughout the period, which continues to the present. This is most evident in the plot of the inverse of the cumulative percent count; the roughly linear decline reflects a continuous addition of new engines to the population.

Figure 4 shows a different presentation of the same information; here, the bar chart illustrates the population of engines within or exceeding the given TSN Index interval as of the end of October 2006. (The percentage decrement is the proportion of engines at that TSN Index level.) Clearly, our interpretation of engine removal lifetime data needs to reflect the difference in the number of engines that have achieved a specific TSN Index level.

\section{Nonparametric Statistical Analysis of F414 Engine Removals}

Parametric statistical methods exist that account for censoring, but exploration of the data using nonparametric methods is necessary to determine whether and what parametric tools might be appropriate. Lawless (2003) provides a recent, accessible, and comprehensive treatment of nonparametric statistical methods for lifetime data and these were applied to the data for the three classes of reasons for removal.

The most useful for visualizing and understanding the F414 UER data were found to be the life table approach. The first challenge in applying this method was to decide the most appropriate lifetime measure. Generally, neither the TSR 
Figure 3: Distribution of the Accumulated EFH Index for the Total F414 Fleet

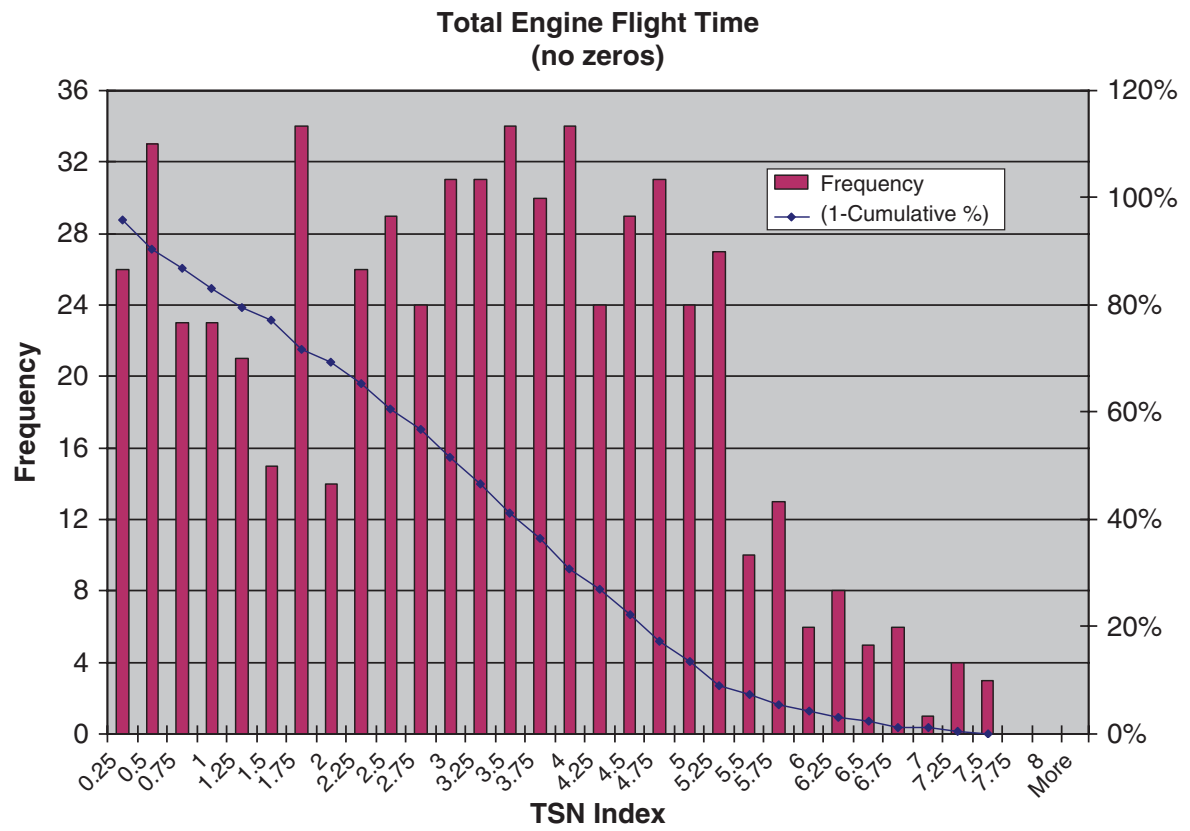

Index nor the TSN Index was suitable; what was appropriate was the TSN Index of the first removal in a given class and the TSN Index difference between each successive pair of removals in the same class.

If a particular engine had not experienced a removal in the class being considered, its total accumulated EFH Index to the end of October 2006 would represent a right-censored data point, a "suspension" in Weibull parametric analysis terms. Similarly, for engines that had experienced a removal in the class being considered, the difference between the TSN Index of the last removal and its total EFH Index at the end of October should be added to the censored data set.
Figure 4: Engine Count Surviving to a Given TSN Index Level

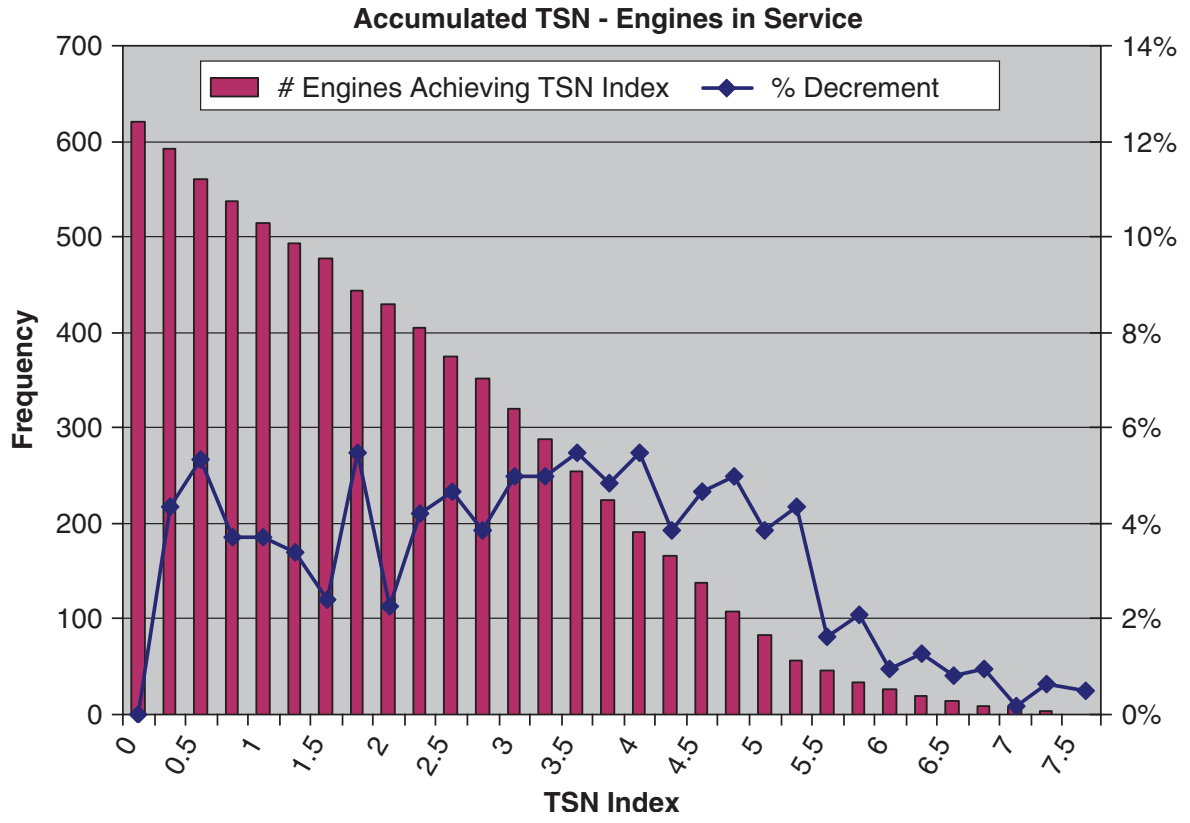


Preparing the data thus required an inspection of the removals plus accumulated EFH Index history for each engine; Table 3 provides a typical example of an individual engine TSN Index history.

This example would contribute two data points for FOD (Code 1) one UER @ 1.495 (this engine suffered FOD before accumulating any flight time after the Code 5 removal and reinstallation) and a subsequent right-censored run of $(2.345-1.495)=0.85 \mathrm{EFH}$ Index up to the end of October 2006.

For the class of Codes 0, 5, and 7, this engine contributes two removals (in this case, with differing codes, the full TSN Indices of 1.495 and 1.859) and the differences of 0.486 $(2.345-1.859)$ and 0.85 to the final installed EFH Index. The last line contributes a right-censored data point at EFH Index 2.345 to all analyses, including the core engine distress (Codes 2, 3, and 4) data set.

Figure 5 illustrates the life table result for FOD using intervals of $0.25 \mathrm{EFH}$ Index, estimates of the hazard rate $[h(t)]$ calculated as the number of removals divided by the population subject to removal, and an approximation to the survivor function $[S(t)]$ obtained incrementally from the hazard rate estimate, i.e., $S_{j}=S_{j-1}-S_{j}\left(h_{j}\right) \sim$ $\mathrm{S}_{\mathrm{j}-1}-\mathrm{S}_{\mathrm{j}-1}\left(\mathrm{~h}_{\mathrm{j}}\right)\left(1-\mathrm{h}_{\mathrm{j}}\right)=\mathrm{S}_{\mathrm{j}-1}\left(1-\mathrm{h}_{\mathrm{j}}+\mathrm{h}_{\mathrm{j}}^{2}\right)$. Standard deviation $(69 \%)$ confidence bounds are shown for both the hazard rate and the survivor function. These increase as the engine population achieving higher lifetimes declines.

Two points are notable, the roughly constant hazard rate (averaging 30\% per EFH Index) after TSN Index 2.0-not unexpected for what is expected to be a constant risk for each takeoff and landing with some average level of mission flight hours. Because FOD is usually detected during preventive maintenance at the $0.6 \mathrm{EFH}$ Index inspections, the $30 \%$ hazard rate implies an $18 \%$ ( $>1$ in 5$)$ chance of an FOD removal at every inspection of an engine after the first inspection.

\begin{tabular}{lc} 
TABLE 3: Typical Engine Removals \\
History \\
\hline Reason for Removal & TSN Index \\
\hline SER & 0.222 \\
SER & 0.802 \\
SER & 1.492 \\
UER - Code 5 & 1.495 \\
UER - Code 1 & 1.495 \\
UER - Code 7 & 1.859 \\
Installed $11 / 2006$ & 2.345 \\
\hline
\end{tabular}

The other is that the survivor function is still about $40 \%$ at the last removal, i.e., $>1 / 3$ of all engines can be expected to achieve a TSN Index of 6.0 without experiencing FOD. Clearly, these results provide useful guidance for maintenance and logistics planning.

Figure 6 shows the result of the same method applied to the removals for various external causes, including maintenance-induced problems and mishaps, i.e., for Codes 0,5 , and 7 . Ignoring the two data points beyond TSN Index 6.0, the removals hazard rate displays behavior similar to that for FOD, a rapid increase above $20 \%$ and relatively constant thereafter. Again, the exogenous causes for many of the failures make this appear reasonable.

Figure 7 investigates the result of the active mitigation efforts expected for this class of removals. While FOD is difficult to counter, the detailed causes affecting this category can be readily addressed through training, changes to maintenance procedures, and relatively rapid configuration upgrades. Many of the configuration changes were introduced over time by scheduled removals or incidental to unscheduled removals for all causes. After Engine 247, a block upgrade incorporated the bulk of these changes, and so this cohort alone was given the same treatment. The difference is startling. The measures adopted were effective in reducing the hazard rate for external causes to below $5 \%$ in all but one interval.

Figure 8, for Codes 2-4, presents the results for core engine distress. In this case the hazard rate 
Figure 5: Estimated Hazard Rate and

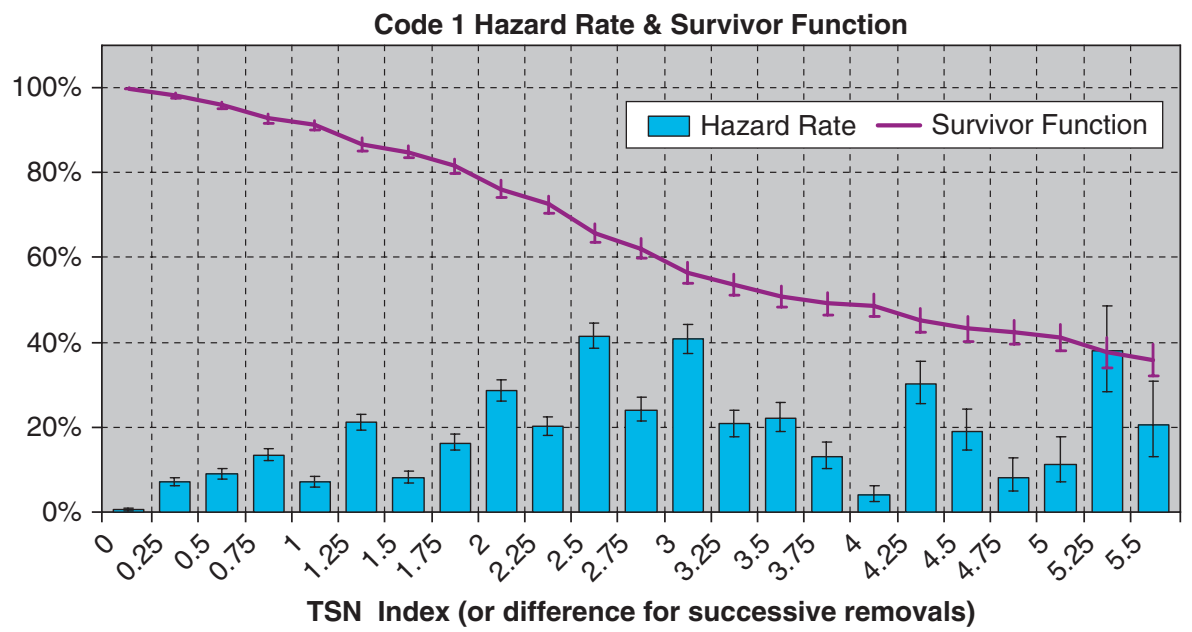

increases slowly through TSN Index 3, and holds at about $20 \%$ thereafter, implying a one in eight chance of an engine removal for core engine distress at every inspection after the fifth one. However, the survivor function estimate suggests that $50 \%$ of all engines will achieve a TSN Index of 6 without a removal for core engine distress.

However, the confidence bounds and the paucity of data make it hard to extrapolate with any degree of confidence beyond TSN Index 6. As a scheduled overhaul is performed about this time, this is not a matter of much practical concern.
Other well-established nonparametric tools, described in Lawless (2003) and other sources, were applied to the Code 2-4 removals to illustrate the changes in engine reliability before and after the block change in configuration at engine \#248 and to analyze competition between preventive maintenance-engines removed as a result of inspections - and failures-engines removed because of mission aborts and IFSD.

The methods used, the Kaplan-Meier Product Limit estimator for the survivor function and the Nelson-Aalen estimator for the cumulative hazard function, and the results are presented in detail in Millar (2007) and Millar et al. (2007).
Figure 6: Estimated Hazard Rate and Survivor Function - Codes 0,5 , and 7

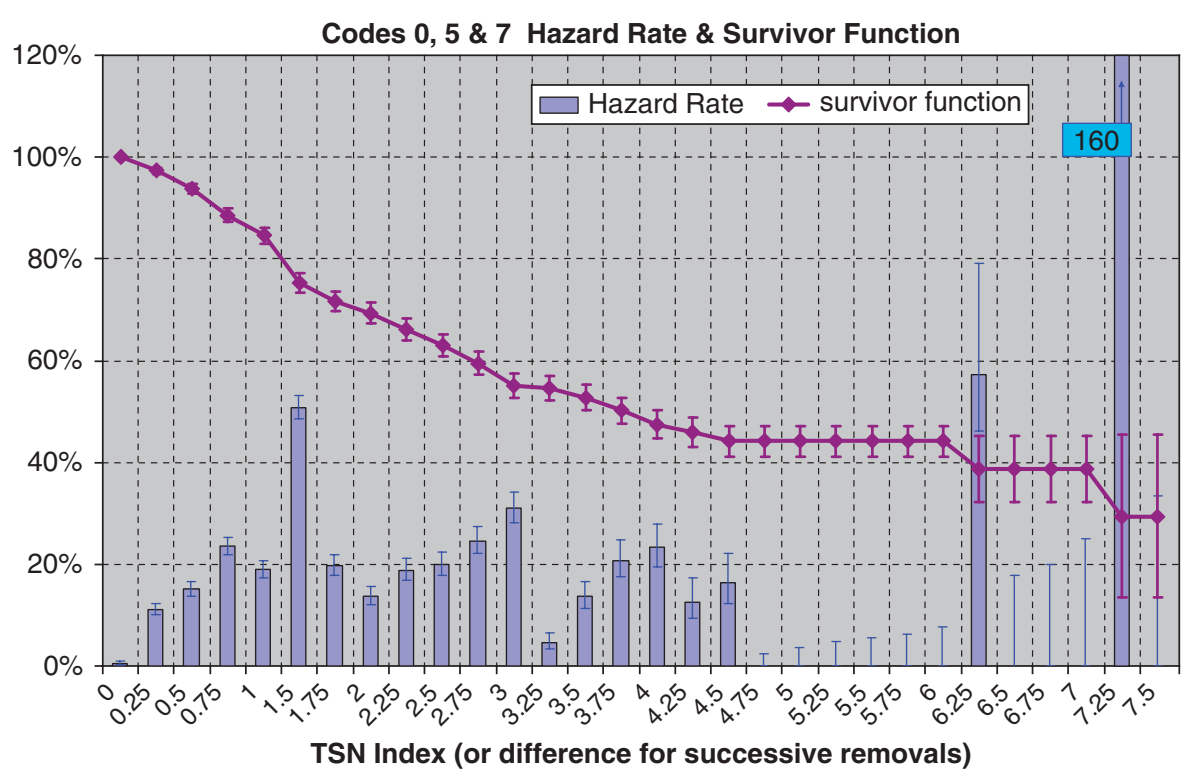




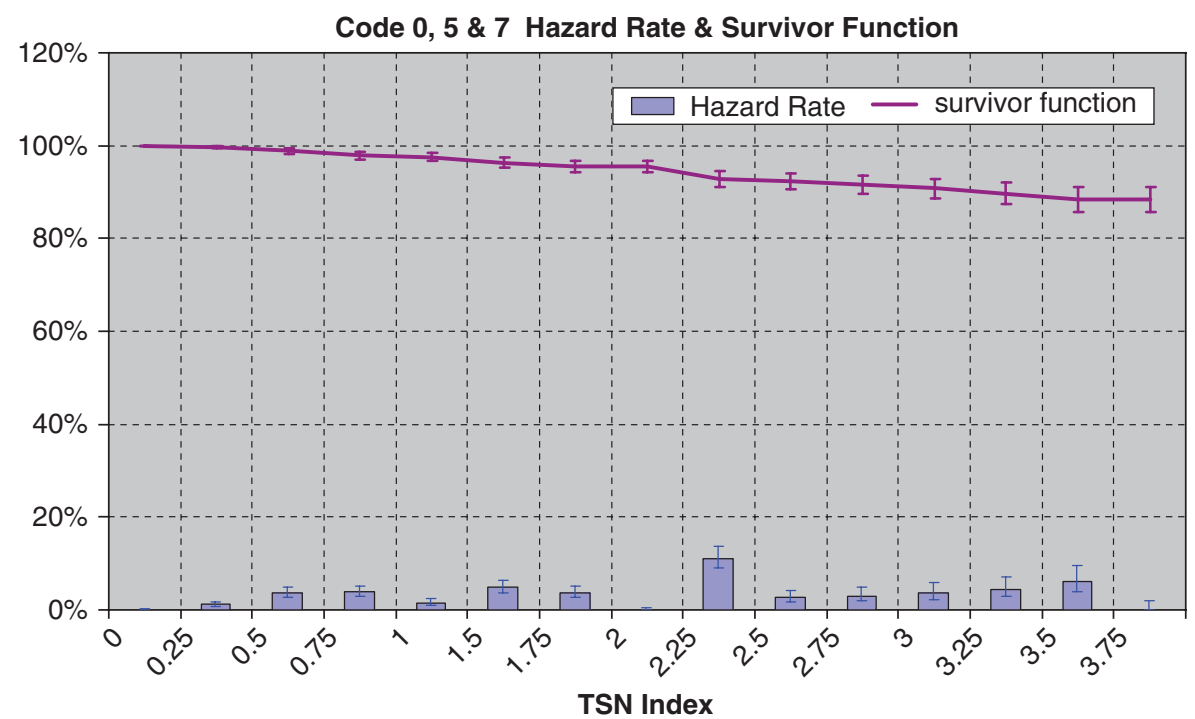

Figure 7: Estimated Hazard Rate and Survivor Function - Codes 0 , 5, and 7 for Engine Numbers 247 and Up.

\section{Conclusions}

The statistical lifetime analysis approach used here adds a crucial and complementary element to the other tools used to monitor engine removals frequency and the reasons for removal-the influence of individual engine TOW on engine removals. Nonparametric statistical lifetime analysis methods applied to this large and complex RDB were surprisingly effective in providing an insight into engine reliability as measured by engine removals at the flight line.

The approach applied provided actionable information on the probability of an O-Level inspection resulting in an engine removal for a given class of reasons for removal. The net result was to show the effectiveness of the overall propulsion system maintenance process (including engine configuration updates) in dynamically managing engine removals to operationally tolerable levels.

Novel treatments of the data to aggregate a tractable number of reasons for removal causes and then generate appropriate lifetime measures appear to have worked well despite heroic assumptions. In particular, the decisions to avoid the complexity of incorporating data on scheduled removals and the competing risk of

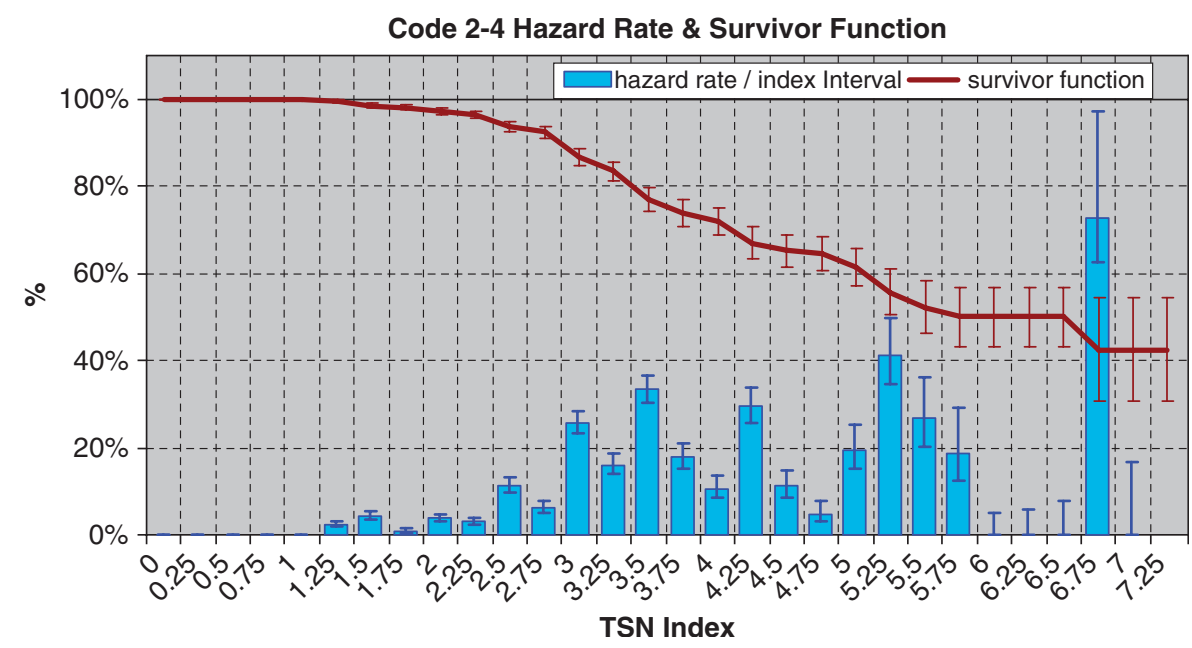

Figure 8: Estimated Hazard Rate and Survivor Function - Code 2-4 Removals 
opportunistic module replacements at the I-Level appear to be justified.

It is hard to conceive that a parametric analysis could add significant actionable information beyond what can be gleaned from these nonparametric procedures - at this level of analysis. The same methods should be of use when applied to removals of WRA providing useful information for maintenance management to address the resultant maintenance workload burden and the operational disruption of mission aborts.

However, a larger task lies ahead: the application of these methods and advanced parametric models for competing risks to the analysis of F414 MDW records of modular removals at the I-Level and component removals and replacements at the depot. This in-depth analysis promises the possibility of comprehensive modeling of F414 engine reliability from the bottom up.

\section{Future Work}

Two Small Business Innovation Research (SBIR) projects have been awarded to develop advanced-automated techniques to mine and analyze the GE MDW RDB in greater depth than was attempted in the research reported here. These efforts include access to I-Level module removal and depot maintenance data.

\section{Hcknowledgment}

The work presented here was made possible by the cooperation and support of GE Aviation and NAVAIR in allowing access to, and publication of, the information contained in the F414 Military Data Warehouse.

\section{References}

Lawless, J.F., Statistical models and methods for lifetime data, 2nd edition, John Wiley \& Sons, Hoboken, NJ, 2003.

Millar, R.C., Non-parametric analysis of a complex propulsion system database, The George Washington University, Washinģton, DC, 2007.

Millar, R.C., T.A. Mazzuchi, and S. Sarkani, "Application of non-parametric statistical methods to reliability da- tabase analysis." SAE AeroTech (Paper 2007-01-3861) Los Angeles, CA, 2007.

Schoch, E.J., "A simulation of the $I 3$ to $D$ repair process and sparing of the F414-GE-400 jet aircraft engine." Naval Postgraduate School, Monterey, CA, 2003.

\section{Huthor Biographies}

Dr. Richard C. Millar is an Associate Professor at the Naval Postgraduate School, stationed at NAS Patuxent River, Maryland. Dr. Millar's career has encompassed over 35 years in gas turbine propulsion \& power research, design and program management, including propulsion system integration for a dozen aircraft development programs.

Dr. Thomas Andrew Mazzuchi is a Professor of Operations Research and Engineering Management and Chair of the Department of Engineering Management and Systems Engineering in the School of Engineering and Applied Science at The George Washington University, Washington, D.C. Dr. Mazzuchi received a B.A. (1978) in Mathematics from Gettysburg College, Gettysburg, PA, a M.S. (1979) and a D.Sc. (1982), both in Operations Research from The George Washington University, Washington D.C. Dr. Mazzuchi s current research interests include reliability growth assessment, software reliability modeling, design and inference in life testing, reliability estimation as a function of operating environment, maintenance inspection policies, and incorporation of expert judgment into reliability and risk analysis.

Shahram Sarkani, Ph.D., P.E., is Professor of Engineering Management and Systems Engineering at The George Washington University. As Faculty Adviser, Academic Director and Head of EMSE Off-Campus Programs since 2001, Professor Sarkani designs and administers off-campus programs on behalf of the Department of Engineering Management and Systems Engineering of GW's School of Engineering and Applied Science. Today EMSE-OCP offers classes in over 25 cities around the U.S. and 
overseas, and enrolls about 1,200 graduate students pursuing master's and doctoral degrees in Systems Engineering and in Engineering Management.

Professor Sarkani has engaged in engineering research, technology development, and engineering education since 1980. He is author of over 150 technical publications and presentations. He joined the GW faculty in 1986, where his administrative appointments include chair of the Civil, Mechanical, and Environmental Engineering Department (1994-1997) and Interim Associate Dean for Research and Development at SEAS (1997-2001).

Professor Sarkani holds the Ph.D. in Civil Engineering from Rice University, and B.S. and M.S. degrees in Civil Engineering from Louisiana State University. He is a Registered Professional Engineer. 